Ann. Geophys., 26, 3501-3509, 2008

www.ann-geophys.net/26/3501/2008/

(C) European Geosciences Union 2008

\title{
Properties of a cusp diamagnetic cavity boundary
}

\author{
B. M. Walsh, T. A. Fritz, and J. Chen \\ Center for Space Physics, Boston University, Boston, MA, USA
}

Received: 25 March 2008 - Revised: 7 October 2008 - Accepted: 15 October 2008 - Published: 12 November 2008

\begin{abstract}
While crossing through the high-altitude dayside cusp on 29 September 1978 and again on 30 October 1978, the ISEE-1 spacecraft observed enhanced energetic particle flux and a depressed and turbulent magnetic field, the signature characteristics of a cusp diamagnetic cavity. As ISEE-1 approached the cavity during each event, a boundary sounding technique was used to measure properties of an emitting boundary. Sounding over multiple energy channels reveals an energy dependent boundary with lower energy particles filling a larger cavity than higher energy particles. Relative motion of the boundary as well as boundary orientation are also measured. The two cusp events were measured at different locations and during different geomagnetic and solar wind conditions; however, they show similar results.
\end{abstract}

Keywords. Magnetospheric physics (Energetic particles, precipitating; Magnetopause, cusp, and boundary layers; Magnetospheric configuration and dynamics)

\section{Introduction}

The magnetospheric cusp was originally modeled as a simple image dipole with focal points of null magnetic field (Chapman and Ferraro, 1931). The geomagnetic field lines threading the cusp were later viewed as a funnel into the magnetosphere allowing solar wind plasma into the cusp region through dayside magnetic merging (Reiff et al., 1977). The interpretation of the cusp region was further developed with the work of Mead (1964); Shabansky and Antonova (1968); Shabansky (1971) and Antonova (1975) who showed the solar wind pressure on the dayside of the geomagnetic field moves the magnetic field minimum to higher latitudes both northward and southward. The off-equatorial minimums cause particle trapping in the high-altitude cusp regions. This paper also uses the observational characteristics

Correspondence to: $\mathrm{B}$. M. Walsh

(bwalsh@bu.edu) of the presence of shocked solar wind plasma (Frank, 1971) and increased density over the adjacent regions to define the cusp.

More recent in situ measurements in the high-altitude dayside cusp from the Polar spacecraft identified cusp diamagnetic cavities (CDCs) defined by a depressed and turbulent magnetic field (Chen et al., 1997, 1998; Chen and Fritz, 1998). Large intensities of cusp energetic particles (CEPs) with energies up to $8 \mathrm{MeV}$ (Chen et al., 1998) are also shown to be present within the CDCs. The depressed magnetic field varies dramatically and rapidly with the magnitude approaching $0 \mathrm{nT}$ during some events. Polar observations have also shown these CDCs to be up to $6 R_{E}$ in size (Fritz et al., 2003). Multispacecraft observations by the Cluster fleet have identified the CDC as a common feature of the exterior cusp. Lavraud et al. (2005) reported Cluster encountering the boundary of a CDC several times before fully entering into the cavity, implying a moving boundary.

The International Sun-Earth Explorer-1 (ISEE-1) was launched 22 October 1977 and traveled in a highly eccentric orbit $\left(1.09 \times 23 R_{E}\right)$ with a period of $\sim 57 \mathrm{~h}$ and an inclination of $28.76^{\circ}$ (Ogilvie et al., 1977). The Medium Energy Particle Experiment (MEPE) onboard the spacecraft measured angular, energy and intensity distributions of energetic electrons and ions (Williams et al., 1978). While measuring in low bit rate, MEPE measured energetic ions with energy bands: 24 $44.5 \mathrm{keV}, 44.5-65.3 \mathrm{keV}, 65.3-95.5 \mathrm{keV}, 95.5-143 \mathrm{keV}, 143-$ $210 \mathrm{keV}, 210-333 \mathrm{keV}, 333-849$, and $849-2081 \mathrm{keV}$. Electrons were measured over the energy bands: $22.5-39 \mathrm{keV}$, $39-75 \mathrm{keV}, 75-120 \mathrm{keV}, 120-189 \mathrm{keV}, 189-302 \mathrm{keV}, 302-$ $477 \mathrm{keV}, 477-756 \mathrm{keV}, 756-1200 \mathrm{keV}$.

As ISEE-1 orbited, it rotated around its spin axis which was normal to the ecliptic plane. The collimator orientation (look direction) of the MEPE detector scanned in polar angle relative to the satellite spin axis and was synchronized with the satellite spin rate. During a $36 \mathrm{~s}$ spin scan, the satellite would rotate 12 times, detecting a range in polar angle from $10^{\circ}$ to $170^{\circ}$. Since the local magnetic field may not be

Published by Copernicus Publications on behalf of the European Geosciences Union. 


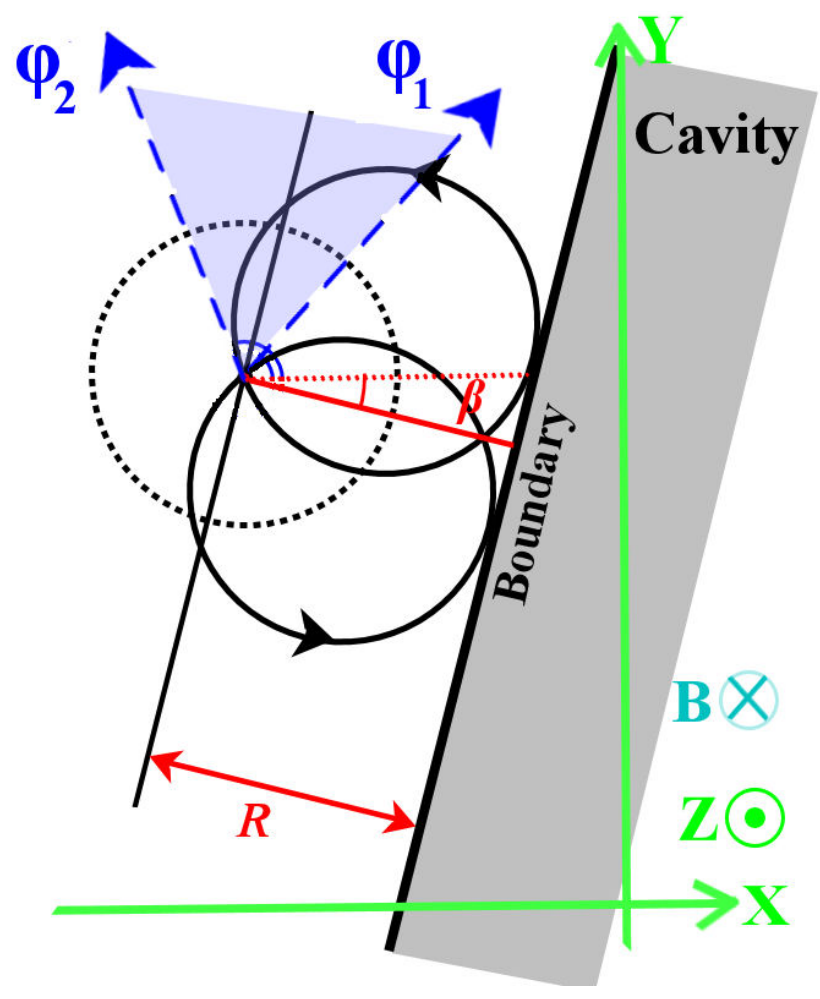

Fig. 1. The center of the dotted circle represents the location of the spacecraft. The two circles drawn with solid black lines represent the path of gyrating ions with the magnetic field aligned in the negative $\mathrm{Z}$ direction. As the location of the spacecraft gets closer to the boundary (gray region), the difference of the angles, $\phi_{2}-\phi_{1}$ (shown in blue) becomes larger. The orientation of the boundary with respect to $\mathrm{X}$ (GSM) is the angle $\beta$ (shown in red), and the distance to the boundary is $R$ (shown in red).

aligned with the spin axis, this range in polar angle during a single spin scan allows particles with a pitch angle (PA) of $90^{\circ}$ to be sampled at 8 (in low bit rate) azimuthal angles around the spin axis: $0^{\circ}, 45^{\circ}, 90^{\circ}, 135^{\circ}, 180^{\circ}, 225^{\circ}, 270^{\circ}$, and $315^{\circ}$ with $0^{\circ}$ and $90^{\circ}$ aligned with the $\mathrm{X}$ and $\mathrm{Y}$ axes in GSE accordingly.

\section{Observations}

The ISEE-1 spacecraft measured enhanced energetic particle flux and a depressed and turbulent local magnetic field consistent with a CDC on 29 September 1978 (Walsh et al., 2007) and 30 October 1978 (Whitaker et al., 2006).

For a CDC that contains both energetic electrons and ions, there are three features that can be identified to measure the boundary of the cavity: (1) The magnetic field strength sharply drops and becomes highly turbulent at the boundary; (2) The presence of energetic electrons (Since the electrons have relatively small gyroradii, the boundary location as measured by the electrons and depressed magnetic field is very similar); (3) Due to their relatively large gyroradii, energetic ions associated with a CDC can be observed for a large distance outside the cavity. This study looks at the boundary as measured by all three signatures but utilizes the large gyroradii of the ions to probe the distance and structure of the CDC.

\subsection{Boundary sounding technique}

Energetic ions were detected using the MEPE and were used to probe the distance to an emitting boundary. As ISEE-1 rotated on its axis, the "look direction" of the detector scanned through all $2 \pi$ radians in azimuthal angle. While ISEE-1 approached an emitting boundary from an area of low flux, particles were detected between azimuthal angles $\phi_{1}$ and $\phi_{2}$ as shown in Fig. 1. The angles $\phi_{1}$ and $\phi_{2}$ represent the look direction of the detector when observing flux enhancements. As the distance between the spacecraft and the boundary decreased, the range in azimuthal angle where enhanced flux was observed $\left(\phi_{2}-\phi_{1}\right)$ became larger. This continued until ISEE-1 was completely inside the cavity and the enhanced flux was observed at all azimuthal angles for particles with a PA of $90^{\circ}$. Previous work has been done sounding boundaries from distances of up to 2 gyroradii from an absorbing boundary (Williams et al., 1979; Fritz and Fanhnensteil, 1982). Whitaker et al. (2007) used ISEE-1 to sound an emitting boundary of a CDC in order to measure its motion and orientation. A similar method to that used by Whitaker et al. (2007) is used in this paper with improvements to the process of identifying the angles $\phi_{1}$ and $\phi_{2}$. This paper also utilizes multiple energy channels to sound particles of a variety of energies to further probe the properties of the cavity.

Through the geometry of the system, the distance $R$ along the boundary normal, between the spacecraft and the emitting boundary is given by Eq. (1).

$R=r_{g}\left(1+\frac{\cos \left(\phi_{2}-\phi_{1}\right)}{2}\right)$

The gyroradius $r_{g}$ is approximated with the local magnetic field strength measured during each $36 \mathrm{~s}$ spin scan. Since the sounding is only conducted as the spacecraft is outside the CDC boundary, the strength of the magnetic field does not change significantly over a $36 \mathrm{~s}$ time period. During the spin scan over the time interval 12:24:54 to $12: 25: 31$ UT on 29 September 1978 as ISEE-1 approached the CDC, the magnetic field strength varied between $128 \mathrm{nT}$ and $125 \mathrm{nT}$, corresponding to gyroradii of $175 \mathrm{~km}$ and $179 \mathrm{~km}$ for a $24 \mathrm{keV}$ ion. Since the changes in the magnetic field strength outside the CDC over a $36 \mathrm{~s}$ time period are small, approximating the strength of the magnetic field with a $36 \mathrm{~s}$ average is appropriate.

The ion flux was measured as the look direction of the MEPE detector scanned through all $4 \pi$ steradians. Figure $2 a$ shows the ion flux as a function of polar angle and azimuthal 


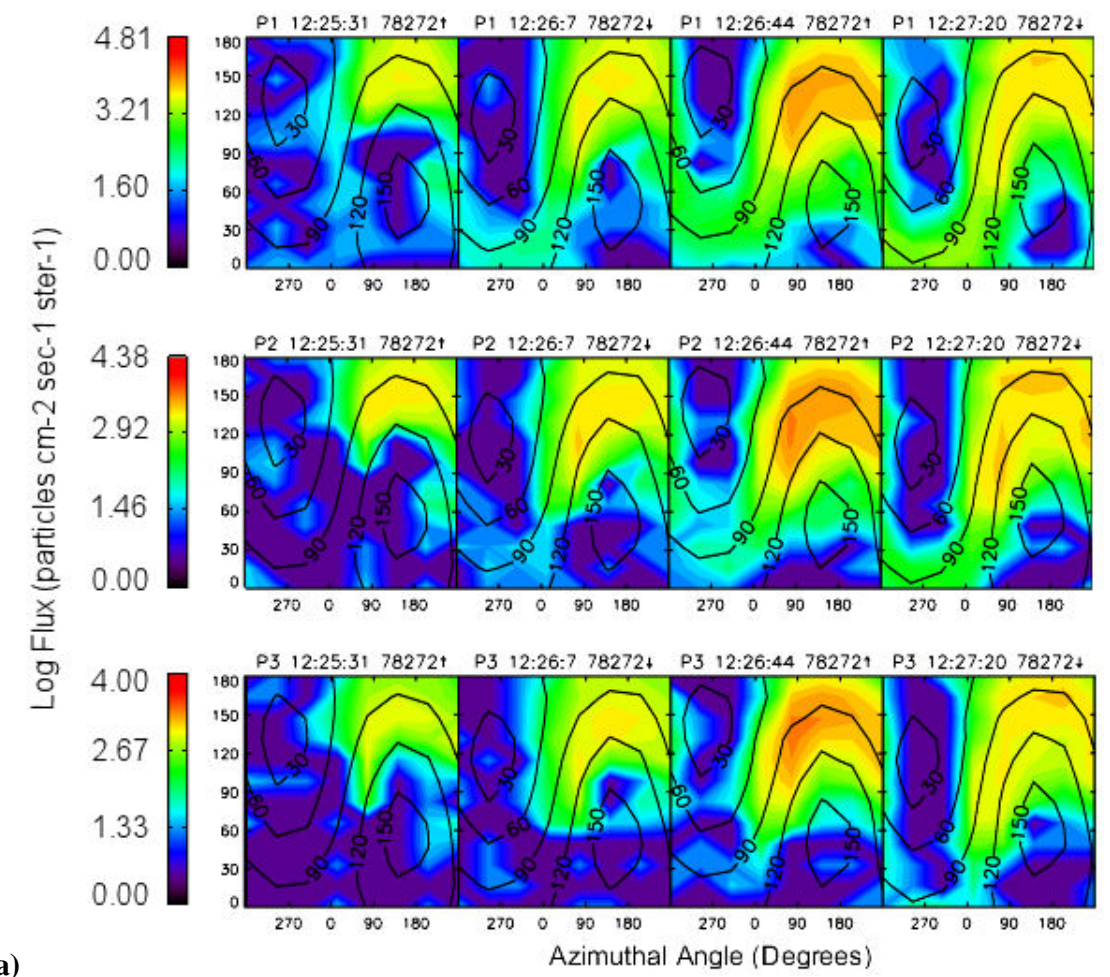

(a)
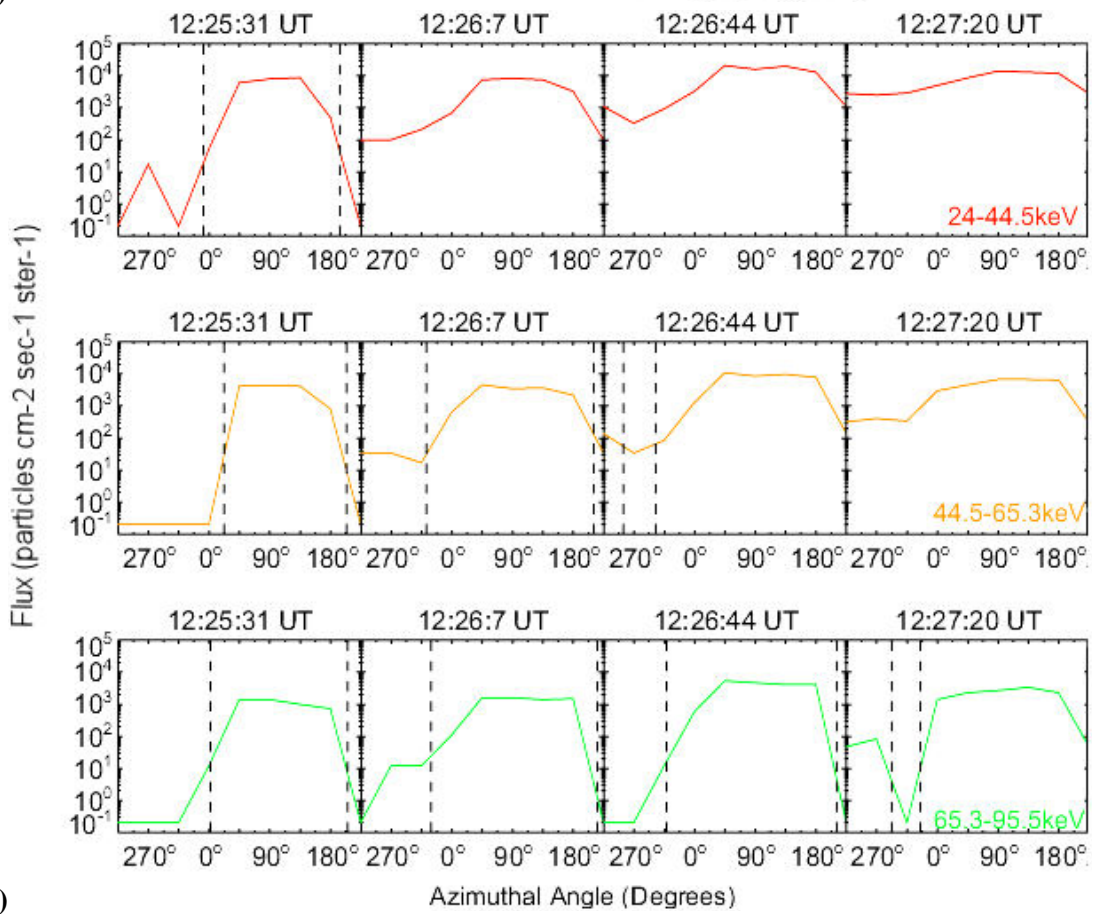

Fig. 2. (a) The plot contains ion flux as a function of azimuthal and polar angle while approaching a CDC boundary on 29 September 1978 during a sounding period. The PA of the particle flux are overlayed on the panels. Each panel represents a $36 \mathrm{~s}$ spin scan. Azimuthal angles of $0^{\circ}$ and $90^{\circ}$ are aligned with the $\mathrm{X}$ and $\mathrm{Y}$ axis in GSE accordingly. Each row presents a different energy channel over the same sounding period, with the lowest energy on top and the highest energy on the bottom. (b) Once again, each row represents the instrument's response to particles of different energies over the same time period, but this plot only presents the flux at a PA of $90^{\circ}$. The vertical dotted lines represent $\phi_{1}$ and $\phi_{2}$. If no values for $\phi_{1}$ and $\phi_{2}$ are plotted, the spacecraft is defined to be within the boundary of the cavity. 


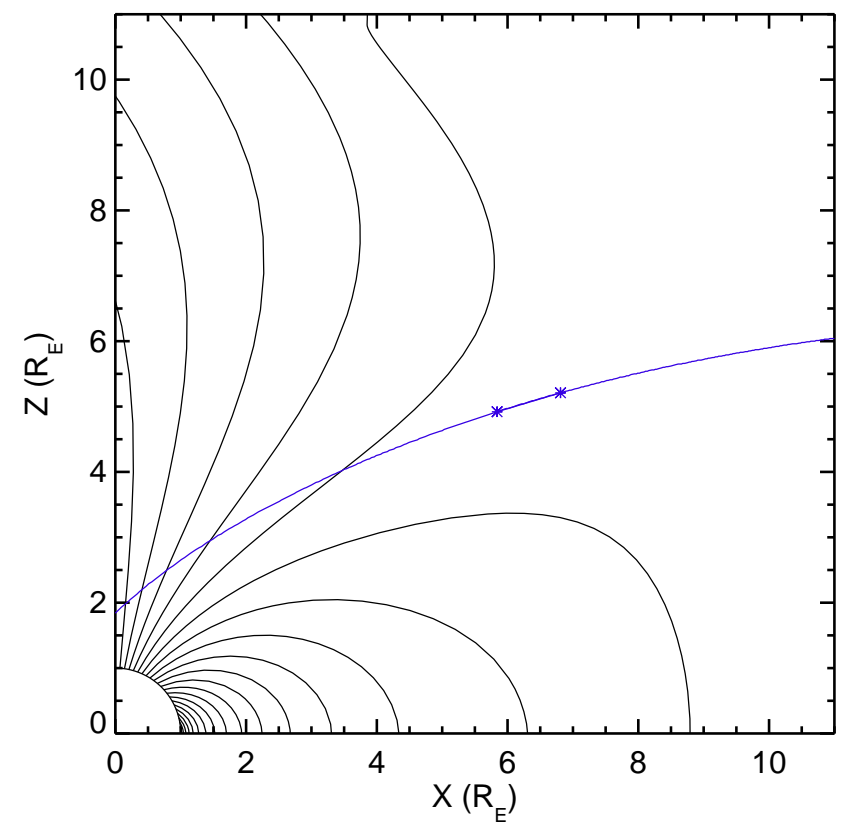

Fig. 3. The magnetic field is simulated with the TS04 model (Tsyganenko and Sitnov, 2005) on 29 September 1978. Trajectory of ISEE-1 in GSM coordinates is in blue. The location of the first and last interactions with the CDC are marked with blue stars at 12:30 and 13:05 UT.

angle of the detector look direction with the PA overlayed. The three rows display the same time period for three different energy channels (24-44.5 keV, 44.5-65.3 keV, and 65.3$95.5 \mathrm{keV}$ ). The flux at a PA of $90^{\circ}$ was isolated to be used for the sounding as shown in Fig. $2 b$ where ion flux at a PA of $90^{\circ}$ was plotted as a function of azimuthal angle over a $36 \mathrm{~s}$ spin scan. Once again, the same time period as used in Fig. $2 \mathrm{a}$ is used and the multiple rows display multiple energy channels while approaching the boundary. The angles $\phi_{1}$ and $\phi_{2}$ plotted in Fig. $2 \mathrm{~b}$ are defined as the limits to the range in azimuthal angle where an enhancement in particle flux is detected. The measurements of $\phi_{1}$ and $\phi_{2}$ were done by identifying where the ion flux was enhanced above 3 sigma of the background level.

The error in the distance measurements is primarily due to the errors induced while identifying $\phi_{1}$ and $\phi_{2}$. Estimating an error of $20^{\circ}$ in measuring both $\phi_{1}$ and $\phi_{2}$, the error in distance to the boundary for a $24 \mathrm{keV}$ proton is roughly $21 \mathrm{~km}$.

The tilt angle of the boundary normal relative to the GSE $\mathrm{X}$-axis is given by Eq. (2). This angle is also illustrated in Fig. 1.

$\beta=\frac{\left(\phi_{1}+\phi_{2}\right)}{2}-90^{\circ}$

The angles $\phi_{1}$ and $\phi_{2}$ in Eq. (2) are defined the same way as above.

\section{Results}

\subsection{September 1978}

On 29 September 1978, ISEE-1 passed through the highaltitude magnetospheric cusp between 12:30 and 13:00 UT at roughly 11:30 MLT. The spacecraft crossed through X=5.78$6.73 R_{E}, \mathrm{Y}=-(1.57-1.54) R_{E}, \mathrm{Z}=4.90-5.19 R_{E}$ in GSM on an outbound pass. The trajectory of the spacecraft in the GSM X-Z plane along with a modeled magnetic field are shown in Fig. 3. During this period, ISEE-1 observed flux enhancements of over two orders of magnitude for both ions and electrons. The ions showed significant flux enhancements up to $210 \mathrm{keV}$ while the electrons showed flux enhancements up to $120 \mathrm{keV}$. Two populations of ions were present: one was trapped along the magnetic field line at a PA of $90^{\circ}$, and the second was flowing from below the spacecraft at a pitch angle of $120^{\circ}$ to $180^{\circ}$. In contrast, only one population of electrons was present, being trapped along the magnetic field line at a PA of $90^{\circ}$. The electron data for this event is displayed in Walsh et al. (2007).

The magnitude of the local magnetic field was both depressed and turbulent. The magnetic field conditions and CEP enhancements indicate a CDC event. ISEE-1 observed several periods during the event where the magnetic field and the particle flux returned close to non-CDC levels. These periods correspond to when the cavity boundary has moved and the spacecraft was no longer within the cavity. This event provides a unique opportunity to perform boundary sounding over several time periods as the spacecraft crossed in and out of the CDC.

The solar wind and IMF conditions were monitored by ISEE-3 near the libration point roughly $200 R_{E}$ upstream from ISEE- 1 and are propagated with a time delay of $20 \mathrm{~min}$ to allow for the travel time between ISEE- 3 and ISEE-1. The solar wind was fast and turbulent, ranging between $820 \mathrm{~km} / \mathrm{s}$ and $780 \mathrm{~km} / \mathrm{s}$. The mass density of the solar wind was low and the dynamic pressure ranged from 1 to $3 \mathrm{nPa}$ during the event. The magnitude of the IMF decreased from $24 \mathrm{nT}$ to $16 \mathrm{nT}$. The IMF components showed a variable and negative $B_{x}$ (antisunward), with a magnitude from $0 \mathrm{nT}$ to $-4 \mathrm{nT}$; duskward $B_{y}$ varying from $18 \mathrm{nT}$ to $14 \mathrm{nT}$ and southward $B_{z}$ decreasing steadily in magnitude from $-15 \mathrm{nT}$ to $-5 \mathrm{nT}$.

\subsubsection{Boundary sounding}

A boundary sounding technique has been used with several energy channels to measure the distance of the CDC to the spacecraft during this event. Distances up to 2 gyroradii or $800 \mathrm{~km}$ for $95.5 \mathrm{keV}$ ions were measured. Figure 4 shows the distance to the boundary as measured through boundary sounding. The event shows five separate periods when the boundary could be sounded as labeled in Fig. 4. The boundary plane itself was approximately perpendicular to GSM/GSE $\mathrm{X}$-axis as identified from the angle $\beta$ found in 


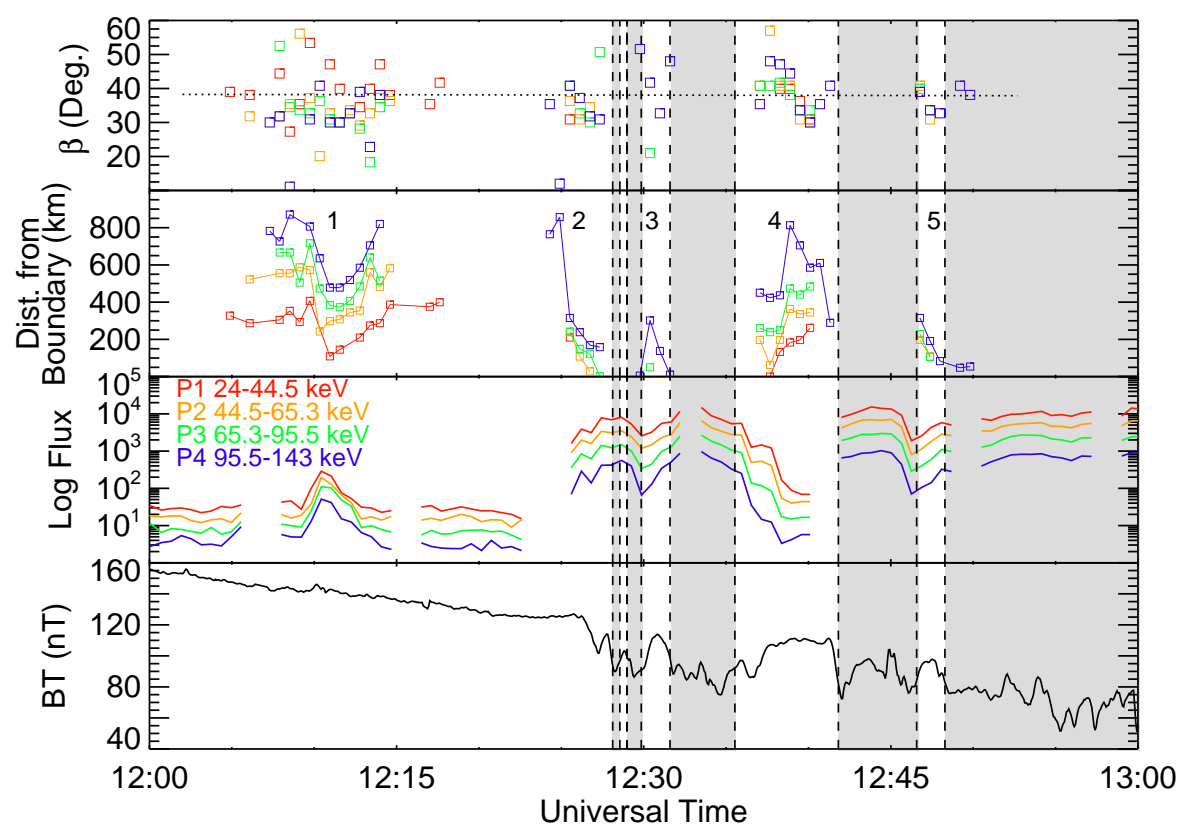

Fig. 4. The first panel is $\beta$ as calculated through Eq. (2) for the first four energy channels $(24-44.5 \mathrm{keV}, 44.5-65.3 \mathrm{keV}, 65.3-95.5 \mathrm{keV}$, 95.5-143 keV) on 29 September 1978. The second panel from the top is the distance between the spacecraft and the emitting boundary as measured from ion sounding. The numbers 1-5 indicate the sounding period. The third panel is ion flux (particles $\mathrm{cm}^{-2} \mathrm{~s}^{-1} \mathrm{ster}^{-1} \mathrm{keV}^{-1}$ ) for the first four energy channels measured by the MEPE. The bottom panel is the local magnetic field strength. The vertical dotted lines and shaded regions show the time periods when the electron flux indicated that the spacecraft had crossed the CDC boundary.

Eq. (2). The angle $\beta$ is relatively constant throughout the event, indicating the orientation of the boundary in the $\mathrm{X}-\mathrm{Y}$ plane does not change.

The first sounding period shows the boundary approached the spacecraft then pulled away at roughly the same speed as the approach. A short period ( 12:15-12:25 UT) between sounding period 1 and 2 shows the distance to the boundary to be more than 2 gyroradii from the spacecraft. The second sounding period shows the boundary approached, and the spacecraft entered the CDC region of the cusp. An entrance is defined by detection of enhanced ion flux at all azimuthal angles during a spin scan for each energy channel observed. The third sounding period $(\sim 12: 30-12: 33$ UT) shows the boundary pulled away from the spacecraft in the positive GSE X direction before it once again approached and the spacecraft crossed the boundary. While the spacecraft was outside the CDC, the magnetic field increased to close to the nonevent level. After the third sounding period, the spacecraft remained within the CDC for roughly 5 min. The fourth sounding period $(\sim 12: 37-12$ :40 UT) shows the boundary moved in the positive GSE X direction away from the spacecraft, and the magnetic field return to a nonevent level. Although it appears the spacecraft reentered the CDC near 12:40 UT, a data gap prevents us from measuring the motion and spacecraft entrance during this period. During the fifth and final sounding period $(\sim 12: 46-12$ :48 UT) the boundary pulled away from the spacecraft in the positive GSE X direction before it once again approached, and the spacecraft reentered the CDC.

The motion of the boundary towards and away from the spacecraft can not be simply explained by a change in the solar wind dynamic pressure or IMF. As noted before, the dynamic pressure remains roughly constant near $2.5 \mathrm{nPa}$ while the IMF $B_{z}$ component increases steadily from $-15 \mathrm{nT}$ to $-5 \mathrm{nT}$ but remains negative. The $B_{y}$ component also remains strongly positive and shows little change.

During the CDC encounter, the boundary approached and retreated from the location of ISEE-1, indicating a moving boundary. To measure the relative velocity of the boundary, a small period was taken to be representative of the boundary motion throughout the event. The period between 12:10 and 12:15 UT was analyzed with the data for P4 (95.5-143 keV). The boundary velocity relative to the spacecraft during this period was $2.4 \mathrm{~km} / \mathrm{s}$ in the X GSM/GSE direction, while the spacecraft itself moved at $3.5 \mathrm{~km} / \mathrm{s}$ in the X GSM/GSE direction relative to the earth. Since the ion data show the boundary approaching and receding in the same direction it approached from, the motion is not consistent with a wave like boundary. 

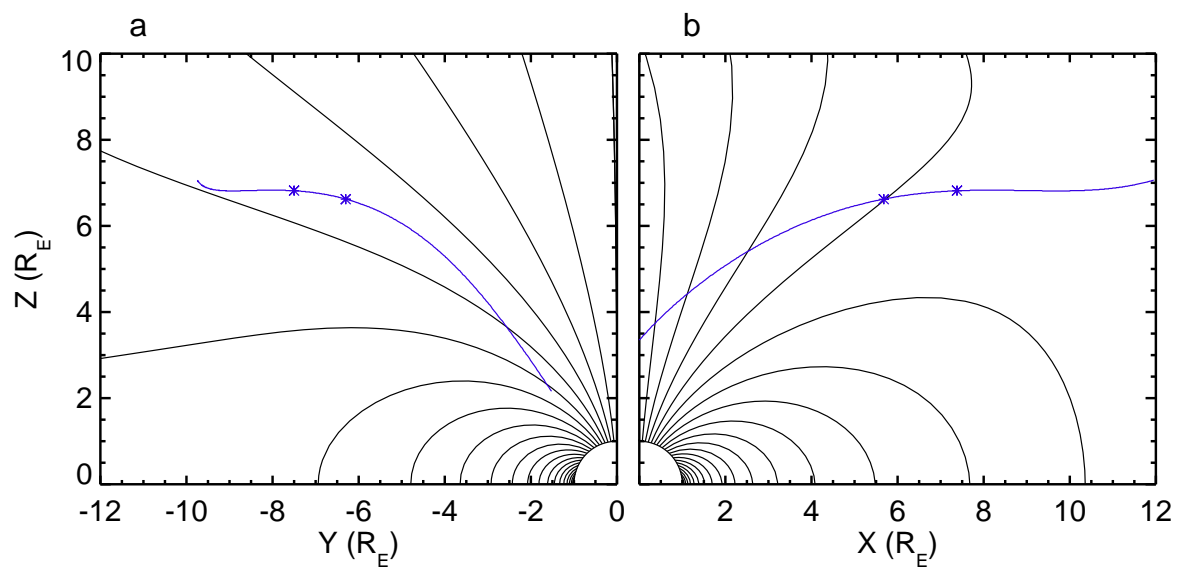

Fig. 5. The magnetic field is simulated with the TS04 model (Tsyganenko and Sitnov, 2005) on 30 October, 1978. Trajectory of ISEE-1 in GSM coordinates is in blue. The location of the first and last interactions with the CDC are marked with blue stars at 16:30 and 18:00 UT.

Table 1. Times at which ISEE-1 entered and exited the CDC as measured by electron flux on 29 September 1978.

\begin{tabular}{ll}
\hline Boundary Transition & Time (UT) \\
\hline Enter 1 & $12: 28: 07$ \\
Exit 1 & $12: 28: 33$ \\
Enter 2 & $12: 28: 59$ \\
Exit 2 & $12: 29: 51$ \\
Enter 3 & $12: 31: 36$ \\
Exit 3 & $12: 35: 32$ \\
Enter 4 & $12: 41: 49$ \\
Exit 4 & $12: 46: 34$ \\
Enter 5 & $12: 48: 17$ \\
Exit 5 & $13: 01: 57$ \\
\hline
\end{tabular}

\subsubsection{Energy dependence}

Measuring the boundary distance with multiple energy channels shows the location of the boundary is energy dependent. Throughout each sounding period, the distance to the emitting boundary as measured by higher energy channels was larger than that measured by lower energy channels. This implies the boundary has an energy gradient in the positive GSE X direction or pointing inward into the CDC. This is also the opposite to what one would expect from a gyroradius effect of a single boundary.

Although the sign of the gradient remains constant throughout the event and at all measured distances into the boundary, the energy gradient itself of the boundary location appears to vary with time. The lack of accurate distance measurements to the boundary prevents an analysis of the variations in the gradient.

An energy dependent boundary is consistent with the effect seen in sounding period 3. During this period, the space- craft crossed through the boundaries and out of the CDC for the higher energy channels (65.3-95.5 keV, 95.5-143 keV) but remained inside the boundary for the two lower energy channels (24.0-44.5 keV, 44.5-65.3 keV). Sounding period 2 shows the same effect, seen in Fig. 2. The lowest energy channel $(24-44.5 \mathrm{keV})$ indicates that the spacecraft entered the cavity three spin scans or $1 \mathrm{~min} 48 \mathrm{~s}$ before the 65.3$95.5 \mathrm{keV}$ particles indicates an entry. The spacecraft is defined as within the cavity when the flux at all azimuthal angles is above three sigma of the background flux.

The presence of energetic electrons were also used as an indicator of the CDC boundary. The times at which ISEE1 crossed the CDC boundary as defined by the energetic electron boundary are shown by the vertical dashed lines in Fig. 4. The location of the electron boundary is closest to that found for the high energy ions. Analyzing multiple electron energy channels shows a boundary in the same location with no apparent energy dependence through the $3 \mathrm{~s}$ cadence provided by the MEPE. Table 1 gives the entry and exit times into the CDC as defined by the trapped electron flux.

\subsection{October 1978}

On 30 October 1978, ISEE-1 passed through the highaltitude dayside northern cusp region from roughly 16:00 to 18:30 UT during an outbound pass. The pass was measured closer to the dawn side than the previous event at roughly $8.5 \mathrm{~h}$ MLT. The spacecraft traveled from $\mathrm{X}=3.9-$ 8.2 $R_{E}, \mathrm{Y}=-(5.0-8.2) R_{E}, \mathrm{Z}=6.0-6.8 R_{E}$ in GSM. The trajectory of the spacecraft in the GSM X-Z and Y-Z plane along with a modeled magnetic field are shown in Fig. 5a and b. A CDC was observed during this period with a depressed and turbulent magnetic field. CEP flux enhancements were observed up to $210 \mathrm{keV}$ for ions while the electrons only showed a small enhancement. Just as with the 29 September 


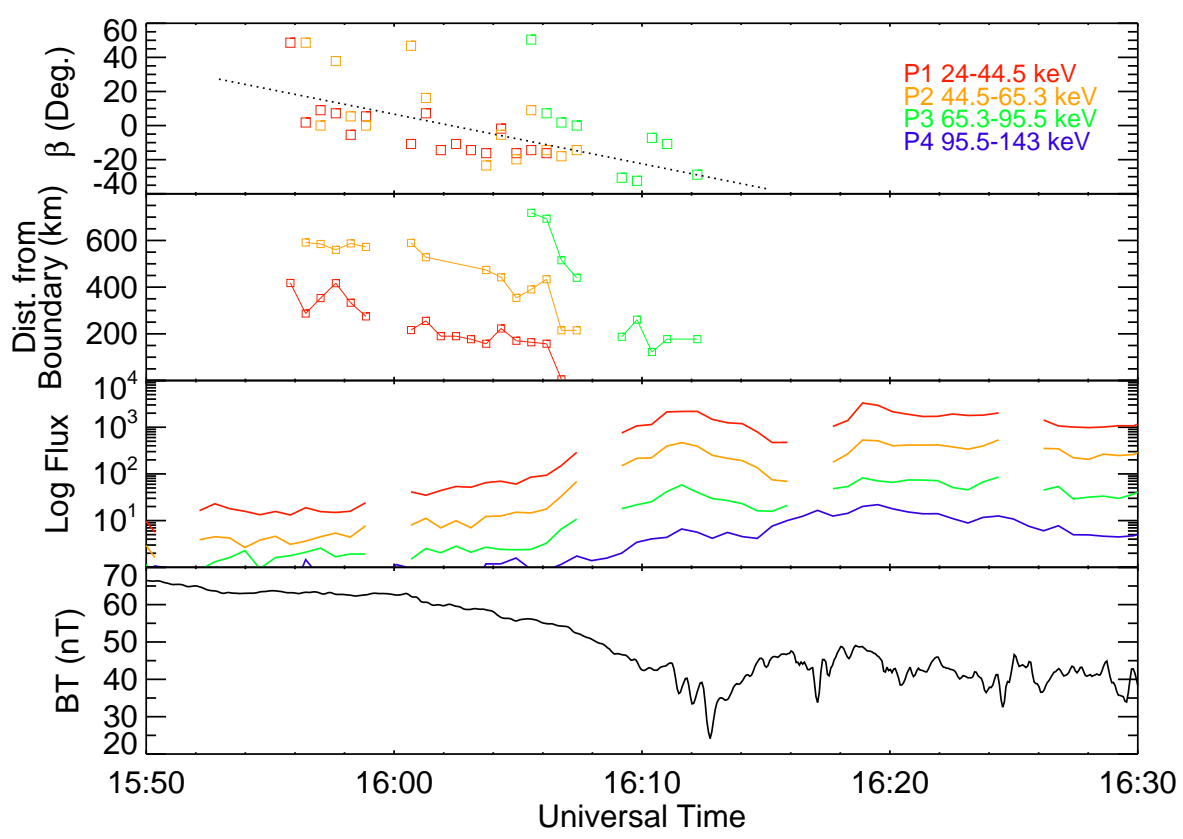

Fig. 6. The first panel is $\beta$ as calculated through Eq. (2) for the first three energy channels $(24-44.5 \mathrm{keV}, 44.5-65.3 \mathrm{keV}$, 65.3-95.5 keV) on 30 October 1978. The second panel from the top is the distance between the spacecraft and the emitting boundary as measured from the ion sounding. The third panel is ion flux (particles $\mathrm{cm}^{-2} \mathrm{~s}^{-1} \mathrm{ster}^{-1} \mathrm{keV}^{-1}$ ) for the first four energy channels. The local magnetic field strength is shown in the bottom panel.

1978 event, the ions had two populations, one trapped at a PA of $90^{\circ}$ and a second flowing from below the spacecraft.

The solar wind and IMF conditions were once again monitored by ISEE- 3 roughly $200 R_{E}$ upstream and close to the libration point. The solar wind was slow and steady $(\sim 380 \mathrm{~km} / \mathrm{s})$. The magnitude of the IMF ranged from 11.5 to $13.0 \mathrm{nT}$. The $B_{x}$ and $B_{z}$ components of the IMF were negative (tailward and southward). The $B_{z}$ component is negative and ranges from $-10 \mathrm{nT}$ to $-8 \mathrm{nT}$.

\subsubsection{Boundary sounding}

A similar sounding technique has been used on a single energy channel for this event by Whitaker et al. (2007), but the event is being revisited to look for trends over multiple energy channels. The same boundary sounding analysis done with the 29 September 1978 observations was repeated with the data from the 30 October 1978 event. A single period from 15:55:49 to 16:06:45 UT as ISEE-1 approached the CDC could be sounded. Since the flux enhancements for this event are not as significant as that from 29 September 1978, the enhancements for the energy band from $95.5-143 \mathrm{keV}$ are too low to use this sounding technique, therefore only the first three energy channels are used for sounding. All three energy channels show the spacecraft approaching the emitting boundary at close to a constant rate until 16:06:09 UT when the boundary approached more quickly and passed over the spacecraft. Taking into account the motion of the spacecraft and the speed at which the boundary is approaching, we interpret this event to show the boundary moving at close to a constant velocity in the positive GSM/GSE X direction. The spacecraft, however is moving at a higher velocity in this direction, so it overtakes the boundary and crosses into the CDC.

The energy dependence of the boundary is consistent with what was seen in the 29 September 1978 event. Once again, there is a clear gradient in energy of the boundary location which points into the CDC. Figure 6 shows the energy dependence and the motion of the boundary relative to the spacecraft. Using the period between 15:58:15 and 16:03:43 UT for P1 (24.0-44.5 keV) to be representative of the boundary motion, the velocity of the boundary relative to the spacecraft is measured to be $0.6 \mathrm{~km} / \mathrm{s}$ moving in the positive GSM/GSE $\mathrm{X}$ direction. Whitaker et al. (2007) measured the boundary to be moving towards the spacecraft at $2.5 \mathrm{~km} / \mathrm{s}$. The discrepancy between the two velocities can be accounted for by the different procedures used for boundary sounding. This study uses an automated search that identifies the areas of enhanced flux as being 3 sigma above the background flux, while Whitaker et al. (2007) did not set a firm limit for when flux is enhanced, therefore, the technique employed by Whitaker et al. (2007) includes larger error. 
The boundary orientation as found through Eq. (2) is at first perpendicular to the GSM/GSE X-axis but rotates by roughly $30^{\circ}$ away from the spacecraft in the X-Y plane shown by the change in angle $\beta$ (Fig. 6). This change in orientation is consistent with the results found by Whitaker et al. (2007).

\section{Discussion}

While crossing through the high-altitude dayside cusp, ISEE1 observations show enhanced energetic particle flux as well as a depressed and turbulent magnetic field, the signature properties of a CDC on 29 September 1978 and 30 October 1978. The two events occur during different geomagnetic and solar wind conditions, yet show the same properties of the CDC. During the 29 September 1978 event, the Dst index was between $-186 \mathrm{nT}$ and $-205 \mathrm{nT}$, indicating a major magnetic storm. The solar wind during this time period was fast $(\sim 800 \mathrm{~km} / \mathrm{s})$ and the IMF had a negative $B_{z}$ component. The Dst index on 30 October 1978 was $\sim-80 \mathrm{nT}$, indicating a moderate magnetic storm. The solar wind speed was significantly lower at $\sim 380 \mathrm{~km} / \mathrm{s}$, and again the IMF had a strong negative $B_{z}$ component. The CDC observed during the 29 September 1978 event also showed greater flux enhancements of energetic particles than the 30 October 1978 event by close to an order of magnitude.

While approaching the CDC, the distance to an emitting boundary was probed through an ion sounding technique. Both events showed an energy dependent boundary where the lower energy ions fill a larger cavity than the higher energy ions. If the cavity boundary was independent of energy, an approaching spacecraft would observe more energetic particles before lower energy ones due to their larger gyroradii. In both the 29 September 1978 and 30 October 1978 event, the boundary sounding technique detected the lowest energy channels first followed by more energetic ones, indicating an energy dependent boundary.

This result describes a new property of the CDC and is an indicator as to the acceleration source for these energetic particles. Walsh et al. (2007) and Whitaker et al. (2006, 2007) investigated these two events and provided evidence pointing towards a local acceleration source. During both events, the ion PA distributions indicated ions were flowing away from the earth. The energetic particles also showed no timeenergy dispersion as would be expected from particles that are accelerated elsewhere and drift to the cusp. Both of these characteristics indicate the particles are accelerated locally.

These observations do not require a specific energization mechanism, but an energy dependent boundary indicates an acceleration source with a gradient in the energization mechanism. Many of the energized particles are trapped along magnetic field lines threading through the CDC. Since the $\mathrm{CDC}$ is larger for lower energy particles than higher energy particles, a local source must cover a larger region or be more efficient for low energy acceleration than for high energy acceleration to populate the energy gradient in the CDC boundary.

\section{Conclusions}

On 29 September 1978 and 30 October 1978, ISEE-1 crossed through a CDC while traveling on an outbound pass through the high-latitude, dayside magnetospheric cusp. Inside the CDC, the spacecraft measured a depressed and turbulent local magnetic field as well as an increased flux of energetic particles. While approaching the CDC, a boundary sounding technique was used to probe the distance to the boundary as well as the boundary properties. Results from the two events were consistent showing a boundary motion in the X GSM/GSE direction with a velocity between $0 \mathrm{~km} / \mathrm{s}$ and $2.4 \mathrm{~km} / \mathrm{s}$ traveling towards and away from the spacecraft. Both events also showed an energy dependent emitting boundary to the CDC. An energy dependent boundary implies the lower energy particles fill a larger cavity than the higher energy particles. Due to the local nature of the source, an energy dependent boundary also indicates a source with an acceleration mechanism that is active over a larger region or more efficient for low energy particles than it is for high energy particles.

Acknowledgements. We gratefully acknowledge use of the ISEE-1 magnetic field data (C. Russell: PI). This research was supported in part by the NASA grant NNG05GE90G.

Topical Editor I. A. Daglis thanks I. Sandahl and another anonymous referee for their help in evaluating this paper.

\section{References}

Antonova, A. E. and Shabansky, V. P.: Particle and magnetic field in the outer dayside geomagnetosphere, Geomagn. Aeron., 15(2), 297-302, 1975.

Chapman, S. and Ferraro, V. C. A.: A new theory of magnetic storms, J. Geophys. Res., 36, 171-186, 1931.

Chen, J., Fritz, T. A., Sheldon, R. B., Spence, H. E., and Spjeldvik, W. N.: A new, temporarily confined population in the polar cap during the August 27, 1996 geomagnetic field distortion period, Geophys. Res. Lett., 24, 1447-1450, 1997.

Chen, J., Fritz, T. A., Sheldon, R. B., Spence, H. E., Spjeldvik, W. N., Fennell, J. F., Livi, S., Russell, C. T., Pickett, J. S., and Gurnett, D. A.: Cusp energetic particle events: Implications for a major acceleration region of the magnetosphere, J. Geophys. Res., 103(A1), 69-78, 1998.

Chen, J. and Fritz, T. A.: Correlation of cusp MeV helium with turbulent ULF power spectra and its implications, Geophys. Res. Lett., 25, 4113-4116, 1998.

Frank, L. A.: Plasma in the Earth's polar magnetosphere, J. Geophys. Res., 76(22), 5202-5219, 1971.

Fritz, T. A. and Fahnenstiel, S. C.: High Temporal Resolution Energetic Particle Soundings at the Magnetopause on November 8, 1977, Using ISEE 2, J. Geophys. Res., 87, 2125-2131, 1982. 
Fritz, T. A., Chen, J., and Siscoe, G. L.: Energetic ions, large diamagnetic cavities, and Chapman-Ferro cusp, J. Geophys. Res., 108(A1), 1028, doi:10.1029/2002JA009476, 2003.

Lavraud, B., Rème, H., Dunlop, M. W., Bosqued, J.-M., Dandouras, I., Sauvaud, J.-A., Keiling, A., Phan, T. D., Lundin, R., Cargill, P. J., Escoubet, C. P., Carlson, C. W., McFadden, J. P., Parks, G. K., Moebius, E., Kistlet, L. M., Amata, E., Bavassano-Cattaneo, M.B., Korth, A., Klecker, B., and Balogh, A.: Cluster observes the high-altitude cusp region, Surv. Geophys., 26, 135-175, 2005.

Ogilvie, K. W., von Rosenvinge T., and Durney, A. C.: International Sun-Earth Explorer: a three-spacecraft program, Science, 198, 131-138, 1977.

Mead, G. D.: Deformation of the geomagnetic field by the solar wind, J. Geophys. Res., 69, 1181-1195, 1964.

Reiff, P. H., Hill, T. W., and Burch, J. L.: Solar wind plasma injections at the dayside magnetospheric cusp, J. Geophys. Res., 82, 479-491, 1977.

Shabansky, V. P.: Some processes in the magnetosphere, Space Sci. Rev., 12, 299-418, 1971.

Shabansky, V. P. and Antonova, A. E.: Topology of particle drift shells in the Earth's magnetosphere, Geomagn. Aeron., Transl., 8, 844-859, 1968.
Tsyganenko, N. A. and Sitnov, M. I.: Modeling the dynamics of the inner magnetosphere during strong geomagnetic storms, J. Geophys. Res., 110, A03208, doi:10.1029/2004JA010798, 2005.

Walsh, B. M., Fritz, T. A., Lender, N. M., Chen, J., and Whitaker, K. E.: Energetic particles observed by ISEE-1 and ISEE-2 in a cusp diamagnetic cavity on 29 September 1978, Ann. Geophys., 25, 2633-2640, 2007, http://www.ann-geophys.net/25/2633/2007/.

Whitaker, K. E., Chen, J., and Fritz, T. A.: CEP population observed by ISEE 1, Geophys. Res. Lett., 33, L23105, doi:10.1029/2006GL027731, 2006.

Whitaker K. E., Fritz, T. A., Chen, J., and Klida, M.: Energetic particles sounding of the cusp with ISEE-1, Ann. Geophys., 25, 1175-1182, 2007, http://www.ann-geophys.net/25/1175/2007/.

Williams, D. J., Keppler, E., Fritz, T. A., Wilken, B., and Wibberenz, G.: The ISEE 1 and 2 Medium Energy Particles Experiment, IEEE Trans. Geosci. Electron., 16, 270-280, 1978.

Williams, D. J.: Magnetopause characteristics inferred from threedimensional energetic particle distributions, J. Geophys. Res., 84, 101-104, 1979. 